\title{
Introduction to Formal Preference Spaces
}

\author{
Eliza Niewiadomska \\ Institute of Mathematics \\ University of Białystok \\ Akademicka 2, 15-267 Białystok \\ Poland
}

\author{
Adam Grabowski \\ Institute of Informatics \\ University of Białystok \\ Akademicka 2, 15-267 Białystok \\ Poland
}

Summary. In the article the formal characterization of preference spaces [1] is given. As the preference relation is one of the very basic notions of mathematical economics 9], it prepares some ground for a more thorough formalization of consumer theory (although some work has already been done - see [17]). There was an attempt to formalize similar results in Mizar, but this work seems still unfinished [18].

There are many approaches to preferences in literature. We modelled them in a rather illustrative way (similar structures were considered in [8]): either the consumer (strictly) prefers an alternative, or they are of equal interest; he/she could also have no opinion of the choice. Then our structures are based on three relations on the (arbitrary, not necessarily finite) set of alternatives. The completeness property can however also be modelled, although we rather follow 2 . which is more general [12. Additionally we assume all three relations are disjoint and their set-theoretic union gives a whole universe of alternatives.

We constructed some positive and negative examples of preference structures; the main aim of the article however is to give the characterization of consumer preference structures in terms of a binary relation, called characteristic relation 10, and to show the way the corresponding structure can be obtained only using this relation. Finally, we show the connection between tournament and total spaces and usual properties of the ordering relations.

MSC: 91B08 03B35

Keywords: preferences; preference spaces; social choice

MML identifier: PREFER_1, version: 8.1.02 5.19.1189

The notation and terminology used in this paper have been introduced in the following articles: [3], [13, [14, [1], [7], [15], 4], [5], [8], 19], 21], 20], 16], and [6]. 


\section{Preliminaries}

Let $X, Y, Z$ be sets. We say that $X, Y$, and $Z$ are mutually disjoint if and only if

(Def. 1) (i) $X$ misses $Y$, and

(ii) $Y$ misses $Z$, and

(iii) $X$ misses $Z$.

Now we state the proposition:

(1) Let us consider a set $A$. Then $\emptyset, A$, and $\emptyset$ are mutually disjoint.

Let us observe that every set which is 2-element is also non empty.

Now we state the propositions:

(2) Let us consider sets $a, b$. Suppose $a \neq b$. Then $\{\langle a, a\rangle,\langle b, b\rangle\} \neq\{\langle a$, $a\rangle,\langle a, b\rangle,\langle b, a\rangle,\langle b, b\rangle\}$.

(3) Let us consider a 2-element set $A$ and elements $a, b$ of $A$. If $a \neq b$, then $A=\{a, b\}$.

(4) Let us consider a 2-element set $A$. Then there exist elements $a, b$ of $A$ such that

(i) $a \neq b$, and

(ii) $A=\{a, b\}$.

(5) Let us consider a non trivial set $A$. Then there exist elements $a, b$ of $A$ such that $a \neq b$.

(6) Let us consider sets $x_{1}, x_{2}, x_{3}, x_{4}$. Then $\left(\left\{x_{1}\right\} \cup\left\{x_{2}\right\}\right) \cup\left\{x_{3}, x_{4}\right\}=$ $\left\{x_{3}, x_{1}, x_{2}, x_{4}\right\}$.

(7) Let us consider sets $a, b$. Suppose $a \neq b$. Then $\{\langle a, a\rangle,\langle b, b\rangle\}$ misses $\{\langle a$, $b\rangle,\langle b, a\rangle\}$.

(8) Let us consider a 2-element set $A$ and elements $a, b$ of $A$. Suppose $a \neq b$. $\operatorname{Then~}_{A}=\{\langle a, a\rangle,\langle b, b\rangle\}$. The theorem is a consequence of (3).

(9) Let us consider elements $a, b$ and a binary relation $R$. Suppose $R=\{\langle a$, $b\rangle\}$. Then $R^{\smile}=\{\langle b, a\rangle\}$.

(10) Let us consider sets $a, b$. Then $a \neq b$ if and only if $\{\langle a, b\rangle\}$ misses $\{\langle a$, $a\rangle,\langle b, b\rangle\}$. Proof: If $a \neq b$, then $\{\langle a, b\rangle\}$ misses $\{\langle a, a\rangle,\langle b, b\rangle\}$.

(11) Let us consider a non empty set $X$, a binary relation $R$ on $X$, and elements $x, y$ of $X$. Suppose $\langle x, y\rangle \notin R^{\mathrm{c}}$. Then $\langle x, y\rangle \in R$.

(12) Let us consider a non empty set $X$ and a binary relation $R$ on $X$. Then $R \cap\left(R^{\smile}\right)^{\mathrm{c}}, R \cap R^{\smile}$, and $R^{\mathrm{c}} \cap\left(R^{\smile}\right)^{\mathrm{c}}$ are mutually disjoint.

(13) Let us consider binary relations $P, R$. If $P$ misses $R$, then $P^{\smile}$ misses $R^{\smile}$.

Let us consider a non empty set $X$ and a binary relation $R$ on $X$. Now we state the propositions: 
(14) $\quad R=\left(\left(\left(R^{\smile}\right)^{\mathrm{c}}\right)^{\smile}\right)^{\mathrm{c}}$.

(15) $\quad R^{\smile}=\left(\left(R^{\mathrm{c}}\right)^{\smile}\right)^{\mathrm{c}}$.

(16) $\quad\left(\left(R^{\smile}\right)^{\mathrm{c}}\right)^{\smile}=R^{\mathrm{c}}$.

\section{Properties of Binary Relations}

Let $X$ be a set. Observe that there exists an order in $X$ which is connected and linear order.

Now we state the propositions:

(17) Let us consider a non empty set $X$ and a total reflexive binary relation $R$ on $X$. Then $R^{\smile}$ is total.

(18) Let us consider a non empty set $X$ and a total binary relation $R$ on $X$. Then field $R=X$.

Let us consider a binary relation $R$. Now we state the propositions:

(19) $R$ is irreflexive if and only if for every element $x$ such that $x \in$ field $R$ holds $\langle x, x\rangle \notin R$.

(20) $R$ is symmetric if and only if for every elements $x, y$ such that $\langle x, y\rangle \in R$ holds $\langle y, x\rangle \in R$.

Now we state the propositions:

(21) Let us consider a set $X$ and a binary relation $R$ on $X$. Then $R \cap R \smile$ is symmetric.

(22) Let us consider a binary relation $R$. Then $R$ is asymmetric if and only if for every elements $x, y$ such that $\langle x, y\rangle \in R$ holds $\langle y, x\rangle \notin R$. Proof: If $R$ is asymmetric, then for every elements $x, y$ such that $\langle x, y\rangle \in R$ holds $\langle y, x\rangle \notin R$ by [19, (15)]. If for every elements $x, y$ such that $\langle x, y\rangle \in R$ holds $\langle y, x\rangle \notin R$, then $R$ is asymmetric.

(23) Let us consider elements $a, b$. If $a \neq b$, then $\{\langle a, b\rangle\}$ is asymmetric. The theorem is a consequence of (22). Proof: Set $R=\{\langle a, b\rangle\}$. For every elements $x, y$ such that $\langle x, y\rangle \in R$ holds $\langle y, x\rangle \notin R$.

(24) Let us consider a non empty set $X$ and a binary relation $R$ on $X$. Then $R \cap\left(R^{\smile}\right)^{\mathrm{c}}$ is asymmetric. The theorem is a consequence of (22).

Let us consider a non empty set $X$ and a total reflexive binary relation $R$ on $X$. Now we state the propositions:

(25) $R \cap R^{\smile}$ is reflexive.

(26) $R \cap R^{\smile}$ is total.

Now we state the propositions:

(27) Let us consider elements $a, b$. Suppose $a \neq b$. Then $\{\langle a, b\rangle,\langle b, a\rangle\}$ is irreflexive and symmetric. The theorem is a consequence of (20). Proof: Reconsider $R=\{\langle a, b\rangle,\langle b, a\rangle\}$ as a binary relation. For every elements $x$, 
$y$ such that $\langle x, y\rangle \in R$ holds $\langle y, x\rangle \in R$. For every element $x$ such that $x \in$ field $R$ holds $\langle x, x\rangle \notin R$.

(28) Let us consider a non empty set $X$, a total binary relation $R$ on $X$, and a binary relation $S$ on $X$. Then $R \cup S$ is total.

(29) Let us consider a non empty set $X$ and a total reflexive binary relation $R$ on $X$. Then $R^{\mathrm{c}} \cap\left(R^{\smile}\right)^{\mathrm{c}}$ is irreflexive and symmetric. The theorem is a consequence of (11) and (20). Proof: For every elements $x, y$ such that $\langle x, y\rangle \in R^{\mathrm{c}} \cap\left(R^{\smile}\right)^{\mathrm{c}}$ holds $\langle y, x\rangle \in R^{\mathrm{c}} \cap\left(R^{\smile}\right)^{\mathrm{c}}$ by [6, (87)].

(30) Let us consider a set $X$ and a binary relation $R$ on $X$. If $R$ is symmetric, then $R^{\mathrm{c}}$ is symmetric. The theorem is a consequence of (11) and (20). PROOF: For every elements $x, y$ such that $\langle x, y\rangle \in R^{\mathrm{c}}$ holds $\langle y, x\rangle \in R^{\mathrm{c}}$ by [19, (15)], [16, (23)].

(31) Let us consider an element $X$ and a binary relation $R$. Then $R$ is antisymmetric if and only if for every elements $x, y$ such that $\langle x, y\rangle,\langle y, x\rangle \in R$ holds $x=y$. Proof: If $R$ is antisymmetric, then for every elements $x, y$ such that $\langle x, y\rangle,\langle y, x\rangle \in R$ holds $x=y$ by [19, (15)].

(32) Let us consider a set $A$ and an asymmetric binary relation $R$ on $A$. Then $R \cup \mathrm{id}_{A}$ is antisymmetric. The theorem is a consequence of (22) and (31). PROOF: For every elements $x, y$ such that $\langle x, y\rangle,\langle y, x\rangle \in R \cup \operatorname{id}_{A}$ holds $x=y$.

(33) Let us consider an element $X$ and a binary relation $R$. Then $R$ is connected if and only if for every elements $x, y$ such that $x \neq y$ and $x, y \in$ field $R$ holds $\langle x, y\rangle \in R$ or $\langle y, x\rangle \in R$.

(34) Let us consider a binary relation $R$. Then $R$ is connected if and only if field $R \times$ field $R=\left(R \cup R^{\smile}\right) \cup$ id $_{\text {field } R}$.

(35) Let us consider a set $A$ and an asymmetric binary relation $R$ on $A$. Then $R$ misses $R^{\smile}$. The theorem is a consequence of (22). Proof: For every elements $x, y,\langle x, y\rangle \notin R \cap R^{\smile}$.

(36) Let us consider binary relations $R, P$. If $R$ misses $P$ and $P$ is symmetric, then $R^{\smile}$ misses $P$. The theorem is a consequence of (13).

Let us consider a set $X$ and an asymmetric binary relation $R$ on $X$. Now we state the propositions:

(37) $R$ misses $\operatorname{id}_{X}$.

(38) $R \cdot R$ misses $\operatorname{id}_{X}$.

Let $X$ be a set and $R$ be a binary relation on $X$. The functor $\operatorname{SymCl} R$ yielding a binary relation on $X$ is defined by the term

(Def. 2) $R \cup R^{\smile}$.

Let $R$ be a total binary relation on $X$. Note that $\mathrm{SymCl} R$ is total.

Let $R$ be a binary relation on $X$. One can verify that $\operatorname{SymCl} R$ is symmetric. 


\section{Preference Structures}

We consider pure preference structures which extend 1-sorted structures and are systems

$$
\text { 〈a carrier, a preference relation〉 }
$$

where the carrier is a set, the preference relation is a binary relation on the carrier.

We consider preference-indifference structures which extend pure preference structures and alternative relational structures and are systems

〈a carrier, a preference relation, an alternative relation〉

where the carrier is a set, the preference relation and the alternative relation are binary relations on the carrier.

We consider preference structures which extend preference-indifference structures, relational structures, and pure preference structures and are systems

〈a carrier, a preference relation, an alternative relation, an internal relation〉

where the carrier is a set, the preference relation and the alternative relation and the internal relation are binary relations on the carrier.

Let us note that there exists a preference-indifference structure which is non empty and strict and there exists a preference-indifference structure which is empty and strict and there exists a pure preference structure which is non empty and strict and there exists a pure preference structure which is empty and strict and there exists a preference-indifference structure which is non empty and strict and there exists a preference structure which is non empty and strict.

Let $X$ be a preference structure. We say that $X$ is preference-like if and only if

(Def. 3) (i) the preference relation of $X$ is asymmetric, and

(ii) the alternative relation of $X$ is a tolerance of the carrier of $X$, and

(iii) the internal relation of $X$ is irreflexive and symmetric, and

(iv) the preference relation of $X$, the alternative relation of $X$, and the internal relation of $X$ are mutually disjoint, and

(v) $\left((\right.$ the preference relation of $\left.X) \cup(\text { the preference relation of } X)^{\smile}\right) \cup$ the alternative relation of $X) \cup$ the internal relation of $X=\nabla_{\alpha}$, where $\alpha$ is the carrier of $X$.

Let $X$ be a set. The functor PrefSpace $X$ yielding a strict preference structure is defined by the term

(Def. 4) $\left\langle X, \emptyset_{X, X}, \nabla_{X}, \emptyset_{X, X}\right\rangle$. 
Let $A$ be a non empty set. Observe that PrefSpace $A$ is non empty and preference-like and there exists a preference structure which is non empty, strict, and preference-like.

A preference space is a preference-like preference structure. Note that every preference structure which is empty is also preference-like and PrefSpace $\emptyset$ is empty and preference-like and there exists a preference space which is empty.

Let $A$ be a trivial non empty set. Let us observe that PrefSpace $A$ is trivial.

Let us observe that PrefSpace $A$ is non empty and preference-like.

\section{Constructing Examples}

Let $A$ be a set. The functor IdPrefSpace $A$ yielding a strict preference structure is defined by

(Def. 5) (i) the carrier of $i t=A$, and

(ii) the preference relation of $i t=\emptyset$, and

(iii) the alternative relation of $i t=\mathrm{id}_{A}$, and

(iv) the internal relation of $i t=\emptyset$.

Let $A$ be a non trivial set. Let us observe that IdPrefSpace $A$ is non preferencelike.

Let $A$ be a 2-element set and $a, b$ be elements of $A$.

The functor PrefSpace $(A, a, b)$ yielding a strict preference structure is defined by

(Def. 6) (i) the carrier of $i t=A$, and

(ii) the preference relation of $i t=\{\langle a, b\rangle\}$, and

(iii) the alternative relation of $i t=\{\langle a, a\rangle,\langle b, b\rangle\}$, and

(iv) the internal relation of $i t=\emptyset$.

Now we state the proposition:

(39) Let us consider a 2-element set $A$ and elements $a, b$ of $A$. If $a \neq b$, then $\operatorname{PrefSpace}(A, a, b)$ is preference-like. The theorem is a consequence of (8), (10), (9), (3), (6), and (23).

Let $A$ be a non empty set and $a, b$ be elements of $A$.

The functor IntPrefSpace $(A, a, b)$ yielding a strict preference structure is defined by

(Def. 7) (i) the carrier of $i t=A$, and

(ii) the preference relation of $i t=\emptyset$, and

(iii) the alternative relation of $i t=\{\langle a, a\rangle,\langle b, b\rangle\}$, and

(iv) the internal relation of $i t=\{\langle a, b\rangle,\langle b, a\rangle\}$.

Now we state the proposition: 
(40) Let us consider a 2-element set $A$ and elements $a, b$ of $A$. Suppose $a \neq b$. Then IntPrefSpace $(A, a, b)$ is non empty and preference-like. The theorem is a consequence of (8), (7), (3), and (27).

\section{Characteristic Relation of a Preference Space}

Let $P$ be a preference-indifference structure. The functor CharRel $P$ yielding a binary relation on the carrier of $P$ is defined by the term

(Def. 8) (The preference relation of $P) \cup($ the alternative relation of $P$ ).

We say that $P$ is PI-preference-like if and only if

(Def. 9) (i) the preference relation of $P$ is asymmetric, and

(ii) the alternative relation of $P$ is a tolerance of the carrier of $P$, and

(iii) (the preference relation of $P) \cap($ the alternative relation of $P$ ) $=\emptyset$, and

(iv) ((the preference relation of $\left.P) \cup(\text { the preference relation of } P)^{\smile}\right) \cup$ the alternative relation of $P=\nabla_{\alpha}$,

where $\alpha$ is the carrier of $P$.

Observe that there exists a non empty strict preference-indifference structure which is PI-preference-like and there exists an empty strict preferenceindifference structure which is PI-preference-like.

Let us consider a non empty preference-indifference structure $P$. Now we state the propositions:

(41) Suppose $P$ is PI-preference-like. Then the preference relation of $P=$ CharRel $P \cap\left((\text { CharRel } P)^{\smile}\right)^{\mathrm{c}}$.

(42) Suppose $P$ is PI-preference-like. Then the alternative relation of $P=$ CharRel $P \cap(\text { CharRel } P)^{\smile}$.

Let us consider a non empty preference structure $P$. Now we state the propositions:

(43) Suppose $P$ is preference-like.

Then the preference relation of $P=\operatorname{CharRel} P \cap\left((\operatorname{CharRel} P)^{\smile}\right)^{\mathrm{c}}$.

(44) Suppose $P$ is preference-like.

Then the alternative relation of $P=\operatorname{CharRel} P \cap(\operatorname{CharRel} P)^{\smile}$.

(45) Suppose $P$ is preference-like.

Then the internal relation of $P=(\text { CharRel } P)^{\mathrm{c}} \cap\left((\text { CharRel } P)^{\smile}\right)^{\mathrm{c}}$. 


\section{Generating Preference Space from Arbitrary (Characteristic) RELATION}

Let $X$ be a set and $R$ be a binary relation on $X$. The functor $\operatorname{Aux}(R)$ yielding a binary relation on $X$ is defined by the term

(Def. 10) $\operatorname{SymCl}\left(\left(R \cap\left(R^{\smile}\right)^{\mathrm{c}} \cup\left(R \cap\left(R^{\smile}\right)^{\mathrm{c}}\right)^{\smile}\right) \cup R \cap R^{\smile}\right)^{\mathrm{c}}$.

Now we state the proposition:

(46) Let us consider a non empty set $X$ and a binary relation $R$ on $X$. Then $\left(\left(R \cap\left(R^{\smile}\right)^{\mathrm{c}} \cup\left(R \cap\left(R^{\smile}\right)^{\mathrm{c}}\right)^{\smile}\right) \cup R \cap R^{\smile}\right) \cup \operatorname{Aux}(R)=\nabla_{X}$.

Let us consider a non empty set $X$ and a total reflexive binary relation $R$ on $X$. Now we state the propositions:

(47) $\operatorname{Aux}(R)=\left(R^{\smile}\right)^{\mathrm{c}} \cap R^{\mathrm{c}} \cup\left(R^{\mathrm{c}}\right)^{\smile} \cap\left(R^{\mathrm{c}} \cup R^{\smile}\right)$.

(48) $R \cap\left(R^{\smile}\right)^{\mathrm{c}}$ misses $\operatorname{Aux}(R)$.

(49) $\operatorname{Aux}(R)$ is irreflexive and symmetric.

Let $X$ be a non empty set and $R$ be a total reflexive binary relation on $X$. One can check that $\operatorname{Aux}(R)$ is irreflexive and symmetric.

Let us consider a non empty set $X$ and a total reflexive binary relation $R$ on $X$. Now we state the propositions:

(50) $R \cap R^{\smile}$ misses $\operatorname{Aux}(R)$.

(51) $R \cap\left(R^{\smile}\right)^{\mathrm{c}}, R \cap R^{\smile}$, and $\operatorname{Aux}(R)$ are mutually disjoint.

Let $X$ be a set and $P$ be a binary relation on $X$. The functor CharPrefSpace $P$ yielding a strict preference structure is defined by

(Def. 11) (i) the carrier of $i t=X$, and

(ii) the preference relation of it $=P \cap\left(P^{\smile}\right)^{\mathrm{c}}$, and

(iii) the alternative relation of $i t=P \cap P^{\smile}$, and

(iv) the internal relation of $i t=\operatorname{Aux}(P)$.

Now we state the proposition:

(52) Let us consider a non empty set $A$ and a total reflexive binary relation $R$ on $A$. Then CharPrefSpace $R$ is preference-like. The theorem is a consequence of (24), (46), (51), (26), and (21).

Let $X$ be a non empty set and $P$ be a binary relation on $X$. Let us observe that CharPrefSpace $P$ is non empty.

Let $P$ be a total reflexive binary relation on $X$. Let us note that CharPrefSpace $P$ is preference-like. 


\section{Flat Preference Spaces}

Let $P$ be a preference structure. We say that $P$ is flat if and only if

(Def. 12) (i) the alternative relation of $P=\mathrm{id}_{\alpha}$, and

(ii) there exists an element $a$ of $P$ such that the preference relation of $P=\{a\} \times(($ the carrier of $P) \backslash\{a\})$ and the internal relation of $P=(($ the carrier of $P) \backslash\{a\}) \times(($ the carrier of $P) \backslash\{a\})$,

where $\alpha$ is the carrier of $P$.

Now we state the proposition:

(53) Let us consider a trivial set $A$. Then IdPrefSpace $A=\operatorname{PrefSpace} A$.

Let $A$ be a trivial non empty set. One can check that IdPrefSpace $A$ is non empty and preference-like.

One can check that IdPrefSpace $A$ is flat.

\section{Tournament Preference Spaces}

Let $P$ be a preference structure. We say that $P$ is tournament-like if and only if

(Def. 13) (i) the alternative relation of $P=\mathrm{id}_{\alpha}$, and

(ii) the internal relation of $P=\emptyset$,

where $\alpha$ is the carrier of $P$.

One can check that every preference structure which is empty is also tournament-like and every preference structure which is tournament-like is also void and there exists an empty preference space which is tournament-like and there exists a non empty preference space which is tournament-like.

Now we state the proposition:

(54) Let us consider a non empty preference space $P$. Then $P$ is tournamentlike if and only if CharRel $P$ is connected, antisymmetric, and total. The theorem is a consequence of (33), (32), (35), (34), and (45). Proof: If $P$ is tournament-like, then CharRel $P$ is connected, antisymmetric, and total by [6, (87)]. If CharRel $P$ is connected, total, and antisymmetric, then $P$ is tournament-like by [21, (22)], [19, (23)], [21, (13)].

\section{Total Preference Spaces}

Let $P$ be a preference structure. We say that $P$ is total if and only if

(Def. 14) (i) the preference relation of $P$ is transitive, and

(ii) the alternative relation of $P=\mathrm{id}_{\alpha}$, and 
(iii) the internal relation of $P=\emptyset$, where $\alpha$ is the carrier of $P$.

Let us observe that every preference structure which is total is also void and every preference structure which is total is also tournament-like and PrefSpace $\emptyset$ is total.

Let $A$ be a set. One can verify that IdPrefSpace $A$ is total.

Let $A$ be a trivial non empty set. Let us note that PrefSpace $A$ is total and there exists an empty preference space which is total and there exists a non empty preference space which is total.

Now we state the proposition:

(55) Let us consider a non empty preference space $P$. Then $P$ is total if and only if CharRel $P$ is a connected order in the carrier of $P$. The theorem is a consequence of $(35),(37),(38)$, and (36). Proof: If $P$ is total, then CharRel $P$ is a connected order in the carrier of $P$ by [15, (12)], 211, (13)], [19, (18), (23)]. If CharRel $P$ is a connected order in the carrier of $P$, then $P$ is total by [15, (12)], [21, (13), (1), (22)].

\section{REFERENCES}

[1] Kenneth J. Arrow. Social Choice and Individual Values. Yale University Press, 1963.

[2] Robert J. Aumann. Utility theory without the completeness axiom. Econometrica, 30(3): 445-462, 1962.

[3] Grzegorz Bancerek. Cardinal numbers Formalized Mathematics, 1(2):377-382, 1990.

[4] Grzegorz Bancerek. The ordinal numbers. Formalized Mathematics, 1(1):91-96, 1990.

[5] Czesław Byliński. Partial functions Formalized Mathematics, 1(2):357-367, 1990.

[6] Czesław Byliński. Some basic properties of sets Formalized Mathematics, 1(1):47-53, 1990.

[7] Agata Darmochwał. Finite sets. Formalized Mathematics, 1(1):165-167, 1990.

[8] Klaus E. Grue and Artur Korniłowicz. Basic operations on preordered coherent spaces. Formalized Mathematics, 15(4):213-230, 2007. doi 10.2478/v10037-007-0025-4

[9] Sören Halldén. On the Logic of Better. Lund: Library of Theoria, 1957.

[10] Emil Panek. Podstawy ekonomii matematycznej. Uniwersytet Ekonomiczny w Poznaniu, 2005. In Polish.

[11] Konrad Raczkowski and Paweł Sadowski. Equivalence relations and classes of abstraction Formalized Mathematics, 1(3):441-444, 1990.

[12] George F. Schumm. Transitivity, preference, and indifference. Philosophical Studies, 52: 435-437, 1987.

[13] Andrzej Trybulec. Domains and their Cartesian products Formalized Mathematics, 1(1): 115-122, 1990.

[14] Andrzej Trybulec. Enumerated sets, Formalized Mathematics, 1(1):25-34, 1990.

[15] Wojciech A. Trybulec. Partially ordered sets Formalized Mathematics, 1(2):313-319, 1990.

[16] Zinaida Trybulec. Properties of subsets. Formalized Mathematics, 1(1):67-71, 1990.

[17] Freek Wiedijk. Arrow's impossibility theorem. Formalized Mathematics, 15(4):171-174, 2007. doi 10.2478/v10037-007-0020-9.

[18] Krzysztof Wojszko and Artur Kuzyka. Formalization of commodity space and preference relation in Mizar. Mechanized Mathematics and Its Applications, 4:67-74, 2005.

[19] Edmund Woronowicz. Relations and their basic properties. Formalized Mathematics, 1 (1):73-83, 1990. 
[20] Edmund Woronowicz. Relations defined on sets Formalized Mathematics, 1(1):181-186, 1990.

[21] Edmund Woronowicz and Anna Zalewska. Properties of binary relations. Formalized Mathematics, 1(1):85-89, 1990.

Received October 7, 2013 